\title{
The Understanding of Emotion among Young Chinese Children
}

Yulong Tang,1 Paul L. Harris,2 Francisco Pons,3 Hong Zou,1 Wenjuan Zhang,4 and Qunxia Xu1

1 Beijing Normal University, Beijing, China

2 Harvard University, Cambridge, MA, USA

3 University of Oslo, Oslo, Norway

4 Wuhan University, Wuhan, Hubei, China 


\section{The Understanding of Emotion among Young Chinese Children}

The development of emotion understanding in young Chinese children was examined. The overall trend in the development of emotion understanding among Chinese preschoolers - as measured by the Test of Emotion Comprehension (TEC) proved similar to that found among preschoolers in Western Europe. However, Chinese children performed better at understanding the distinction between real and apparent emotion and worse at understanding the connection between reminders and emotion. Children's theory of mind and verbal ability were significant predictors of their ability to understand emotion.

Keywords: emotion understanding; culture; preschoolers; Chinese 


\section{Introduction}

The ability to understand emotion is a consistent correlate of several social and behavioral outcomes among children (for a meta-analysis, see Trentacosta \& Fine, 2010), such as greater popularity (Cassidy, Parke, Butkovsky, \& Braungart, 1992; Denham, McKinley, Couchoud, \& Holt, 1990), fewer internalizing behaviors (Fine, Izard, Mostow, Trentacosta, \& Ackerman, 2003), and more prosocial behaviors (Ensor \& Hughes, 2005). Improving children's understanding of emotion is a central objective of many intervention programs for socio-emotional development, both for typically developing (e.g., Greenberg, Kusche, Cook, \& Quamma, 1995) and atypically developing children (e.g., Golan et al., 2010).

Despite being used to assess emotion understanding in a variety of different cultures, the TEC has never been used in China - a major and classic representative of an East-Asian culture. In the present study, we compared children's understanding of emotion in China to that reported for children in three other West European cultures. We also examined the relationship between emotion understanding, verbal ability, and theory of mind.

The organization of emotion understanding in children

Among various models of emotion understanding, the nine components included in the Test of Emotion Comprehension (TEC) have been widely studied and accepted (Pons \& Harris, 2000; Pons, Harris \& de Rosnay, 2004). The nine components are: (1) recognizing facial expressions of emotion; and understanding: (2) the external causes

of emotion; (3) the link between desire and emotion; (4) the link between belief and Tang, Y., Harris, P., Pons, F., Zou, H., Zhang, W., \& Xu, Q. (in press). The Understanding of Emotion among Young Chinese Children. International Journal of Behavioural Development. 
emotion; (5) the relation between reminders and emotion; (6) the regulation of emotion; (7) the hiding of felt emotion; (8) mixed or ambivalent emotions; and (9) moral emotions. The nine components can be organized into a three-phase, developmental framework. The first phase (around 3-5 years) focuses on the understanding of basic of emotions: the recognition of facial expressions, understanding external causes of emotion, and understanding the relationship between desires and emotion. The intermediate phase (around 5-8 years) focuses on more mentalistic aspects of emotion; the relation between reminders and emotion, the connection between beliefs and emotion, and the hiding of felt emotion. The final phase, (around 8-11 years) focuses on reflective processes: the regulation of emotion, mixed emotions, and moral emotions.

\section{Cultural similarities and differences in the understanding of emotion}

Several studies have suggested that the understanding of emotion displays a universal pattern across different cultures. Thus, TEC results from the UK, Brazil, Switzerland, Norway, Denmark, Italy, Germany, and Peru reveal similar age changes

(Bender et al., 2015; Karstad et al., 2016; Molina, Bulgarelli, Henning, \& Aschersleben, 2014; Pons et al., 2004; 2014; Roazzi et al., 2009; Tenenbaum, Visscher, Pons, \& Harris, 2004).

However, findings for the age at which children from different cultural backgrounds understand the hiding of felt emotion have been mixed. In the UK, the USA and Japan, the percentage of 4- and 6-year-olds differentiating between the felt and expressed emotion of the story protagonist was similar across all three cultural Tang, Y., Harris, P., Pons, F., Zou, H., Zhang, W., \& Xu, Q. (in press). The Understanding of Emotion among Young Chinese Children. International Journal of Behavioural Development. 
settings (Gardner, Harris, Ohmoto, \& Hamazaki, 1988; Gross \& Harris, 1988; Harris, Donnelly, Guz, \& Pitt-Watson, 1986). Nevertheless, a study by Joshi and MacLean (1994) produced somewhat different results with Indian children. Consistent with earlier findings, they observed an overall improvement between 4 and 6 years in the differentiation between felt and expressed emotion but 4-year-old girls in India outperformed 4-year-old girls in the UK. Molina et al. (2014) compared the performance of middle-class preschoolers In Italy and Germany with the TEC. The two samples displayed a very similar pattern of development for eight of the nine components of the TEC. However, the two samples clearly differed on the Hiding of Emotion component. Italian children were more likely than German children to understand that felt emotion can be hidden. In summary, the results obtained with the TEC show that the general direction of development is similar but that cultural factors may impact children's understanding of particular components.

In the present study, we examined the development of emotion understanding among young children in China. China has several distinctive cultural characteristics: a long history as a single nation, a vast territory and population united by a common language (almost all people speak Mandarin), an emphasis on deference and respect for authority, and a one party system, with its own definition of democracy and freedom of speech. Given its distinctive history and status, it is appropriate to ask if the development of emotion understanding in China is comparable to the pattern observed in other cultures. Hitherto, the emotion understanding of Mainland Chinese children and European-American children has been compared in only one publication. Tang, Y., Harris, P., Pons, F., Zou, H., Zhang, W., \& Xu, Q. (in press). The Understanding of Emotion among Young Chinese Children. International Journal of Behavioural Development. 
Chinese preschoolers performed worse than European-American preschoolers in understanding the causes of emotion (Wang, 2008). However, performance on this component may not reflect children's broader understanding of emotion.

In this study, we used the TEC to examine the development of emotion understanding in Chinese preschoolers. We predicted that the overall trend in the development of Chinese preschoolers' emotion understanding would be similar to that for West European children. Nevertheless, we anticipated that Chinese children might perform better than European children on the Hiding of Emotion component. We also assessed children's verbal ability and theory of mind performance. In line with past research, we anticipated that both would both be predictors of children's understanding of emotion.

\section{Method}

\section{Participants}

A total of 1924 - to 6-year-olds children was tested: 65 4-year-olds ( $\mathrm{M}=4$; $1, \mathrm{SD}$ $=4$ months, range: $3 ; 6-4 ; 8,29$ female $), 60$ 5-year-olds $(\mathrm{M}=5 ; 3, \mathrm{SD}=3$ months, range: 4;9-5;8, 29 female), and 676 -year-olds $(M=6 ; 5, S D=4$ months, range: 5;9-6;8, 33 female). Children were recruited from a kindergarten in Hangzhou serving predominantly middle-class families $(68.5 \%$ of parents had attended college, $74.6 \%$ of families had an income over 10,000 yuan). For each age group, two classes of children were randomly selected from a total of six classes. Mandarin was the only language spoken by the children Tang, Y., Harris, P., Pons, F., Zou, H., Zhang, W., \& Xu, Q. (in press). The Understanding of Emotion among Young Chinese Children. International Journal of Behavioural Development. 
The local ethical committee of Beijing Normal University approved the study. Before testing, we explained the procedure to the school principal, the teachers and the parents of all participants and received approval from them. Parents also signed a voluntary participation agreement. All participants were informed of their right not to participate and to withdraw at any time.

\section{Measures and Procedure}

\section{Test of Emotion Comprehension}

The Test of Emotion Comprehension (TEC) assesses nine different components of emotion understanding. These components vary in complexity and emerge between 3 and 11 years (see Castro, Cheng, Halberstadt, \& Grühn, 2016 for a recent review). It has been translated into 25 languages at the time of writing. It has shown good test-retest reliability, as well as concurrent, criterion and construct validity (See Pons \& Harris, 2000; Pons et al., 2004 for a full description of the procedure and materials for the TEC).

The Chinese version of the TEC was adapted to a Chinese context based on pilot testing that resulted in two minor changes. The text for one of the illustrations to the External Cause component was revised as follows: "This boy/girl is standing at the bus stop, waiting to take a bus to go to school, just like he/she does every day." The criterion target emotion was "alright". This modification was introduced to prevent children from thinking that the bus was delayed, causing the protagonist to be angry. Second, in the illustration for the Belief component, the fox was replaced by a wolf;

in Chinese culture, the wolf is regarded as the animal that preys on rabbits. The Tang, Y., Harris, P., Pons, F., Zou, H., Zhang, W., \& Xu, Q. (in press). The Understanding of Emotion among Young Chinese Children. International Journal of Behavioural Development. 
administration of the test took from 10 to 15 minutes.

Children received one point for passing any given component. The first two components each included five items. Children received one point if they were correct on at least four of the five items. For the remaining seven components, children received one point if they chose the correct option from four options. Thus, children's total score could range from 0 to 9.

\section{Verbal Ability}

The Core Vocabulary scale of the Wechsler Preschool and Primary Scale of Intelligence-Revised (WPPSI: Chinese version) was used to assess children's verbal ability in Mandarin. Total scores on this subscale could range from 0 to 44 . Administration est took from 2 to 10 minutes, depending on the length of children's response to each test item.

Theory of Mind

The Theory-of-Mind Scale (Chinese version) (Fang, Wellman, Liu, Liu, \& Kang, 2009) was used to assess children's theory of mind. This scale includes five subtasks: diverse desires, diverse beliefs, knowledge-access, false belief, and hidden emotion. Children's total score could range from 0-5. Administration from 8 to 10 minutes.

\section{Procedure}

Children's verbal ability and theory-of-mind understanding were assessed in one session; their emotion understanding was assessed with the TEC in a second session, one week later. Children were tested individually in a quiet area of their kindergarten.

\section{Results}

Tang, Y., Harris, P., Pons, F., Zou, H., Zhang, W., \& Xu, Q. (in press). The Understanding of Emotion among Young Chinese Children. International Journal of Behavioural Development. 
The development of emotion understanding

Children's TEC scores ranged from 0 to $8(\mathrm{M}=4.43, \mathrm{SD}=1.61, \mathrm{n}=192)$. No child succeeded on all nine components. A two-way ANOVA of Age (4-, 5-, 6-year olds) X Gender (boys vs. girls) revealed a significant main effect of Age $(F(2,186)=$ 44.21, $p<0.001, \eta^{2}=0.32$ ) but no significant effect of Gender and no interaction of Age X Gender. As expected, 6-years-olds $(\mathrm{M}=5.55, \mathrm{SD}=1.61)$ performed better than 5-year-olds $(\mathrm{M}=4.35, \mathrm{SD}=1.17)$, and 5-year-olds performed better than 4-year-olds $(\mathrm{M}=3.39, \mathrm{SD}=1.65)$.

Figure 1 shows the percentage of children passing each component as a function of age. Inspection of Figure 1 reveals that performance improved with age for all but two components. Kruskal-Wallis one way analyses confirmed that the number of children passing seven of the components increased with age: Recognition: $\chi^{2}(2)=$ 17.56, $p<0.001$; External cause: $\chi^{2}(2)=34.74, p<0.001$; Desire: $\chi^{2}(2)=38.99$, $p<0.001$; Hiding: $\left.\chi^{2}(2)=9.93, p<0.001\right)$; Belief: $\chi^{2}(2)=20.18, p<0.001$; Reminder: $\chi^{2}(2)=6.30, p<0.05$; and Regulation: $\chi^{2}(2)=20.58, p<0.001$. The percentage of children passing the Mixed and Morality components remained low across all three age groups.

Figure 1 about here

Close inspection of Figure 1 reveals that the nature of these age-based improvements varied across the seven components. With respect to four components (Recognition, External Cause, Desire and Hiding) 4-year-olds showed considerable proficiency, with more than $50 \%$ passing each component. Nevertheless, Tang, Y., Harris, P., Pons, F., Zou, H., Zhang, W., \& Xu, Q. (in press). The Understanding of Emotion among Young Chinese Children. International Journal of Behavioural Development. 
Mann-Whitney $U$ tests also confirmed that there was a significant improvement between 4 and 5 years for each: Recognition $(Z=2.85, p<0.01)$, External Cause $(Z=$ 2.97, $\mathrm{p}<0.01)$, Desire $(\mathrm{Z}=4.20, \mathrm{p}<0.001)$, and Hiding $(\mathrm{Z}=2.42, \mathrm{p}<0.05)$. By contrast, there was no improvement for three of these four components between 5 and 6 years for Recognition $(Z=0.68$, n.s. $)$, Desire $(Z=1.50$, n.s. $)$, and Hiding $(Z=0.36$, n.s.); the understanding of External Cause did improve $(Z=3.23, p<0.01)$.

With respect to three other components (Belief, Reminder, Regulation) fewer 4-year-olds showed proficiency, with no more than 33\% passing any of these components and no significant improvement between 4 and 5 years: Belief $(Z=0.48$, n.s.), Reminder ( $Z=0.30$, n.s. $)$, and Regulation $(Z=0.58$, n.s. $)$. There was, however, a significant improvement between 5 and 6 years: $\operatorname{Belief}(Z=3.51, p<0.001)$, Reminder $(Z=2.24, \mathrm{p}<0.05)$, and Regulation $(Z=4.01, \mathrm{p}<0.001)$.

Finally, as Inspection of Figure 1 confirms, few children of any age performed successfully on the Mixed and Morality components.

In sum, children improve in their understanding of particular components of emotion at different points in the preschool years. An understanding of Recognition, External Cause, Desire and Hiding becomes quite widespread between 4 and 5 years of age. By contrast, the understanding of Belief, Reminder, and Regulation shows little change between 4 and 5 years but increases significantly between 5 and 6 years. The understanding of the Mixed and Morality components remains limited throughout this age period. 


\section{Cultural differences in the understanding of emotion}

To examine potential cultural differences, we rank-ordered the components in terms the percentage of children passing each component. We compared this rank-order to those observed in three other samples: British children (Pons et al., 2003), German children, and Italian children (Molina et al., 2014), as shown in Table 1. The British and German cultures can be regarded as representative of individualistic cultures whereas the Chinese and Italian cultures can be regarded as representative of more collectivist cultures (See Table 1 for the size, age range, and mean age of each of the four samples). Because our sample age range was from 4 to 6 years, we selected only the 3- to 7-year-olds from the total sample of British children.

Table 1 about here

Spearman rho coefficients indicated a strong correlation between the rank-order observed in the Chinese sample and each of the other three samples: British $\rho(9)=$ 0.82; German $\rho(9)=0.92$; Italian $\rho(9)=0.90$. Nevertheless, a close comparison of the rank-order of particular components shows that the groups differed with respect to the relative difficulty of the Reminder and Hiding components. Chinese preschoolers performed better on the Hiding component (rank order of 4) as compared to the Reminder component (rank order of 6). Similarly, the Italian children also performed better on the Hiding component (rank order of 3 ) as compared to the Reminder component (rank order of 5). Both groups differed from the British and German children who performed better on the Reminder component (rank order of 2 and 4 respectively) as compared to the Hiding component (rank order of 6 and 5 Tang, Y., Harris, P., Pons, F., Zou, H., Zhang, W., \& Xu, Q. (in press). The Understanding of Emotion among Young Chinese Children. International Journal of Behavioural Development. 
respectively).

To assess whether the variable ordering of the Hiding and Reminder components disturbed the three-phase ordering identified by Pons et al. (2004), we compared the scores of each participant for phases 1 and 2, and for phases 2 and 3 . If the scores for a later phase are equal to or less than the scores for an earlier phase, then that is consistent with the assumption that mastery of an earlier phase is needed for mastery of a later phase. The results showed that for $94.3 \%$ of the children, their scores for phase 2 were equal to or less than their scores for phase 1 and that for the $92.2 \%$ of children their scores at phase 3 were equal to or less than their scores for phase 2. Thus, although cultural differences do exist, they rarely disrupted the overall three-phase development pattern.

\section{Verbal ability, Theory of mind, and Emotional comprehension}

Table 2 shows the descriptive statistics (means and standard deviations) and Person's correlation coefficients among the TEC scores, verbal ability, Theory-of-Mind scores, and age (with and without controls for age). All correlations were significant and in the theoretically expected directions.

Table 2 about here

To further examine the influence of verbal ability and theory of mind on the understanding of emotion, a multiple regression was performed. Children's age in months was entered as a first step, verbal ability as a second, and theory-of-mind score as a third. Each variable contributed significantly to the prediction of children's among Young Chinese Children. International Journal of Behavioural Development. 
understanding of emotion. In total, the model explained $40.2 \%$ of variance. Age made the greatest contribution, $R^{2}=0.34, F(1,190)=95.86, p<0.001$. Nevertheless, after controlling for age, verbal ability was also a significant predictor at Step $2, R^{2}=0.38$, $F(2,189)=57.36, p<0.001$ (with a unique contribution of 4\%). Finally, at Step 3,

Theory of Mind also had a significant effect, $R^{2}=0.40, F(3,188)=42.20, p<0.001$ (with a unique contribution of $3 \%$ ).

\section{Discussion}

The overall trend in the development of Chinese preschoolers' emotion understanding was similar to that of children in Western Europe (England, Germany and Italy). Confirming earlier findings, children's understanding of several components improved markedly between 4 and 6 years of age. The correlations between the rank-order observed in China and in three European countries were high (between .82 and .92). Nevertheless, children from the two more collectivist cultures (China and Italy) performed worse on the Reminder component than the Hiding component whereas children from the two more individualist cultures (Britain and Germany) showed the reverse pattern. Chen (2009) also observed a relatively slow understanding of the Reminder component among Chinese children (as compared to American children). She speculated that differential patterns of emotion socialization might explain this cultural difference. Chinese mothers talk about past events and experiences less frequently with their children than do American mothers. Talk about past emotional episodes is likely to serve as a trigger for past feelings. Such talk might alert children to the connection between reminders and the re-activation of past Tang, Y., Harris, P., Pons, F., Zou, H., Zhang, W., \& Xu, Q. (in press). The Understanding of Emotion among Young Chinese Children. International Journal of Behavioural Development. 
emotion.

Chinese children performed well on the Hiding Emotion component, echoing previous findings with Italian children (Molina et al., 2014). Arguably, in more individualist cultures, people express their emotions directly and freely. By contrast, in more collectivistic cultures, maintaining group harmony is prioritized. Hence, people in a collectivistic culture may hide their actual feelings in deference to the group. If Chinese children are expected to hide their real feeling in some social situations they are likely to have a more frequent and early experience of the discrepancy between felt and expressed emotion than children growing up in more individualistic countries.

Inspection of the scores for each developmental phase confirmed that there were very few children whose score for a later phase were higher than their score for an earlier phase, suggesting that variation occurs within a phase, and not with respect to the overall the developmental pattern.

The findings of the present study provide further evidence of inter-connections between theory of mind and emotion understanding (Eggum et al., 2011). Weimer, Sallquist, and Bolnick (2012) also found that children with higher scores on theory-of-mind questions performed better on the TEC. Similarly, Chen, Cui, and Wang, (2005) found that Chinese preschoolers' performance on a false belief task predicted their knowledge of a story protagonist's feeling. Our results also confirmed that children's verbal ability predicts their emotion understanding. Children start to talk about emotion at around 2 years old (Harris, de Rosnay, \& Pons, 2016) and previous Tang, Y., Harris, P., Pons, F., Zou, H., Zhang, W., \& Xu, Q. (in press). The Understanding of Emotion among Young Chinese Children. International Journal of Behavioural Development. 
research has indicated that verbal ability is a good predictor of both theory-of-mind understanding and emotion understanding (Astington \& Jenkins, 1999; Cutting \& Dunn, 1999; Pons, Lawson, Harris \& de Rosnay, 2003). Everyday discussion is likely to promote children's understanding of mental states, including emotional states (Cutting \& Dunn, 1999; de Rosnay et al., 2004). The current findings extend this link beween language and emotion understanding to Chinese, Mandarin-speaking children.

In conclusion, we found that the overall trend in the development of emotion understanding among Chinese preschoolers is quite similar to that seen among preschoolers in Western Europe. Nevertheless, we also observed differences in the rank ordering of particular components. Unlike British and German children, Chinese children performed better on the task assessing their understanding of the distinction between real and apparent emotion than on the task assessing their understanding of the connection between memory and emotion. Children's theory of mind and their verbal ability were also significant predictors of their emotion understanding. 


\section{Acknowledgments}

This research was supported by the Ministry of Education for Humanities and Social

Science, China (Grant No. 14JJD190003) and by the China Scholarship Council

(201606040059). We thank Lijuan Tang for help in data-collection. Finally, we are

grateful to the children and teachers at the Xiasha Shiyan Kindergarten, Hangzhou.

Tang, Y., Harris, P., Pons, F., Zou, H., Zhang, W., \& Xu, Q. (in press). The Understanding of Emotion among Young Chinese Children. International Journal of Behavioural Development. 


\section{References}

Astington, J. W., \& Jenkins, J. M. (1999). A longitudinal study of the relation between language and theory-of-mind development. Developmental Psychology, 35, $1311-1320$.

Bender, P., Sømhovd, M., Pons, F., Reinholdt-Dunne, M., \& Esbjørn, B. (2015). The impact of attachment security and emotion dysregulation on anxiety in children and adolescents. Emotional and Behavioral Difficulties, 20, 189-204.

Cassidy, J., Parke, R. D., Butkovsky, L., \& Braungart, J. M. (1992). Family-peer connections - The roles of emotional expressiveness within the family and children's understanding of emotion. Child Development, 63, 603-618.

Castro, V. L., Cheng, Y., Halberstadt, A. G., \& Grühn, D. (2016). EUReKA! A conceptual model of emotion understanding. Emotion Review, 8, 258-268.

Chen, D. (2009). Culture, parent-child conversation, and children's understanding of emotion. (Doctoral dissertation), Graduate School of Education, Harvard University, Cambridge, MA.

Chen, Y., Cui Y., \& Wang, Y. (2005). The theory of mind and emotion understanding of preschoolers: Their development and relationship. Psychological Science, 28, 527-532. (Chinese Journal)

Cutting, A. L., \& Dunn, J. (1999). Theory of mind, emotion understanding, language, and family background: Individual differences and interrelations. Child Development, 70, 853-865.

Tang, Y., Harris, P., Pons, F., Zou, H., Zhang, W., \& Xu, Q. (in press). The Understanding of Emotion among Young Chinese Children. International Journal of Behavioural Development. 
de Rosnay, M., Pons, F., Harris, P. L., \& Morrell, J. M. B. (2004). A lag between understanding false belief and emotion attribution in young children: Relationships with linguistic ability and mothers' mental-state language. British Journal of Developmental Psychology, 22, 197-218.

Denham, S. A., McKinley, M., Couchoud, E. A., \& Holt, R. (1990). Emotional and behavioral predictors of preschool peer ratings. Child Development, 61, $1145-1152$.

Eggum, N. D., Eisenberg, N., Kao, K., Spinrad, T. L., Bolnick, R., Hofer, C., . . . Fabricius, W. V. (2011). Emotion understanding, theory of mind, and prosocial orientation: Relations over time in early childhood. Journal of Positive Psychology, 6, 4-16.

Ensor, R., \& Hughes, C. (2005). More than talk: Relations between emotion understanding and positive behaviour in toddlers. British Journal of Developmental Psychology, 23, 343-363.

Fang, F., Wellman, H., Liu, Y., Liu, G., \& Kang, R. (2009). Longitudinal perspectives: The sequences of theory-of-mind development in Chinese preschoolers. Acta Psychologica Sinica, 41, 706-714.

Fine, S. E., Izard, C. E., Mostow, A. J., Trentacosta, C. J., \& Ackerman, B. P. (2003). First grade emotion knowledge as a predictor of fifth grade self-reported internalizing behaviors in children from economically disadvantaged families. Development and Psychopathology, 15, 331-342.

Gardner, D., Harris, P., Ohmoto, M., \& Hamazaki, T. (1988). Japanese children's Tang, Y., Harris, P., Pons, F., Zou, H., Zhang, W., \& Xu, Q. (in press). The Understanding of Emotion among Young Chinese Children. International Journal of Behavioural Development. 
understanding of the distinction between real and apparent emotion. International Journal of Behavioral Development, 11, 203-218.

Golan, O., Ashwin, E., Granader, Y., McClintock, S., Day, K., Leggett, V., \& Baron-Cohen, S. (2010). Enhancing emotion recognition in children with autism spectrum conditions: An intervention using animated vehicles with real emotional faces. Journal of Autism and Developmental Disorders, 40, 269-279.

Greenberg, M. T., Kusche, C. A., Cook, E. T., \& Quamma, J. P. (1995). Promoting emotional comptence in school-aged children - The effects of the path curriculum. Development and Psychopathology, 7, 117-136.

Gross, D., \& Harris, P. L. (1988). False beliefs about emotion: Children's understanding of misleading emotional displays. International Journal of Behavioral Development, 11, 475-488.

Harris, P. L., de Rosnay, M., \& Pons, F. (2016). Children's understanding of emotion. In Lewis, M., Haviland-Jones, J. M. \& Barrett, L.F. (Eds), Handbook of emotions (4th ed.). New York: Guilford Press.

Harris, P. L., de Rosnay, M., \& Pons, F. (2005). Language and children's understanding of mental states. Current Directions in Psychological Science, 14, 69-73.

Harris, P. L., Donnelly, K., Guz, G. R., \& Pitt-Watson, R. (1986). Children's understanding of the distinction between real and apparent emotion. Child Development, 57, 895-909.

Joshi, M. S., \& MacLean, M. (1994). Indian and English children's understanding of the distinction between real and apparent emotion. Child Development, 65, Tang, Y., Harris, P., Pons, F., Zou, H., Zhang, W., \& Xu, Q. (in press). The Understanding of Emotion among Young Chinese Children. International Journal of Behavioural Development. 
$1372-1384$.

Karstad, S. B., Vikan, A., Berg-Nielsen, T. S., de Lucena Moreira, P., de Abreu, E. L., \& Rique, J. (2016). Young Brazilian children's emotion understanding: A comparison within and across cultures. Journal of Educational and Developmental Psychology, 6, 113-124

Molina, P., Bulgarelli, D., Henning, A., \& Aschersleben, G. (2014). Emotion understanding: A cross-cultural comparison between Italian and German preschoolers. European Journal of Developmental Psychology, 11, 592-607.

Pons, F., de Rosnay, M., Bender, P., Doudin, P.-A., Harris, P., \& Gimenez-Dasi, M. (2014). The impact of abuse and learning difficulties on emotion understanding in late childhood and early adolescence. Journal of Genetic Psychology, 175, 301-307.

Pons, F., \& Harris, P. L. (2000). Test of emotion comprehension: TEC: University of Oxford.

Pons, F., Harris, P. L., \& de Rosnay, M. (2004). Emotion comprehension between 3 and 11 years: Developmental periods and hierarchical organization. European Journal of Developmental Psychology, 1, 127-152.

Pons, F., Lawson, J., Harris, P., \& de Rosnay, M. (2003). Individual differences in children's emotion understanding: Effects of age and language. Scandinavian Journal of Psychology, 44, 347-3531.

Roazzi, A., Dias, M., Minervino, C., Roazzi, M., \& Pons, F. (2009). Children's comprehension of emotion. A cross-cultural investigation. In D. Elizur \& E. Yaniv Tang, Y., Harris, P., Pons, F., Zou, H., Zhang, W., \& Xu, Q. (in press). The Understanding of Emotion among Young Chinese Children. International Journal of Behavioural Development. 
(Eds.) Theory construction and multivariate analysis: Applications of Facet Approach (109-123). Tel-Aviv: FTA Publications.

Tenenbaum, H. R., Visscher, P., Pons, F., \& Harris, P. L. (2004). Emotional understanding in Quechua children from an agro-pastoralist village. International Journal of Behavioral Development, 28, 471-478.

Trentacosta, C. J., \& Fine, S. E. (2010). Emotion knowledge, social competence, and behavior problems in childhood and adolescence: A meta-analytic review. Social Development, 19, 1-29.

Wang, Q. (2008). Emotion knowledge and autobiographical memory across the preschool years: A cross-cultural longitudinal investigation. Cognition, 108, 117-135.

Weimer, A. A., Sallquist, J., \& Bolnick, R. R. (2012). Young children's emotion comprehension and theory of mind understanding. Early Education and Development, 23, 280-301. 


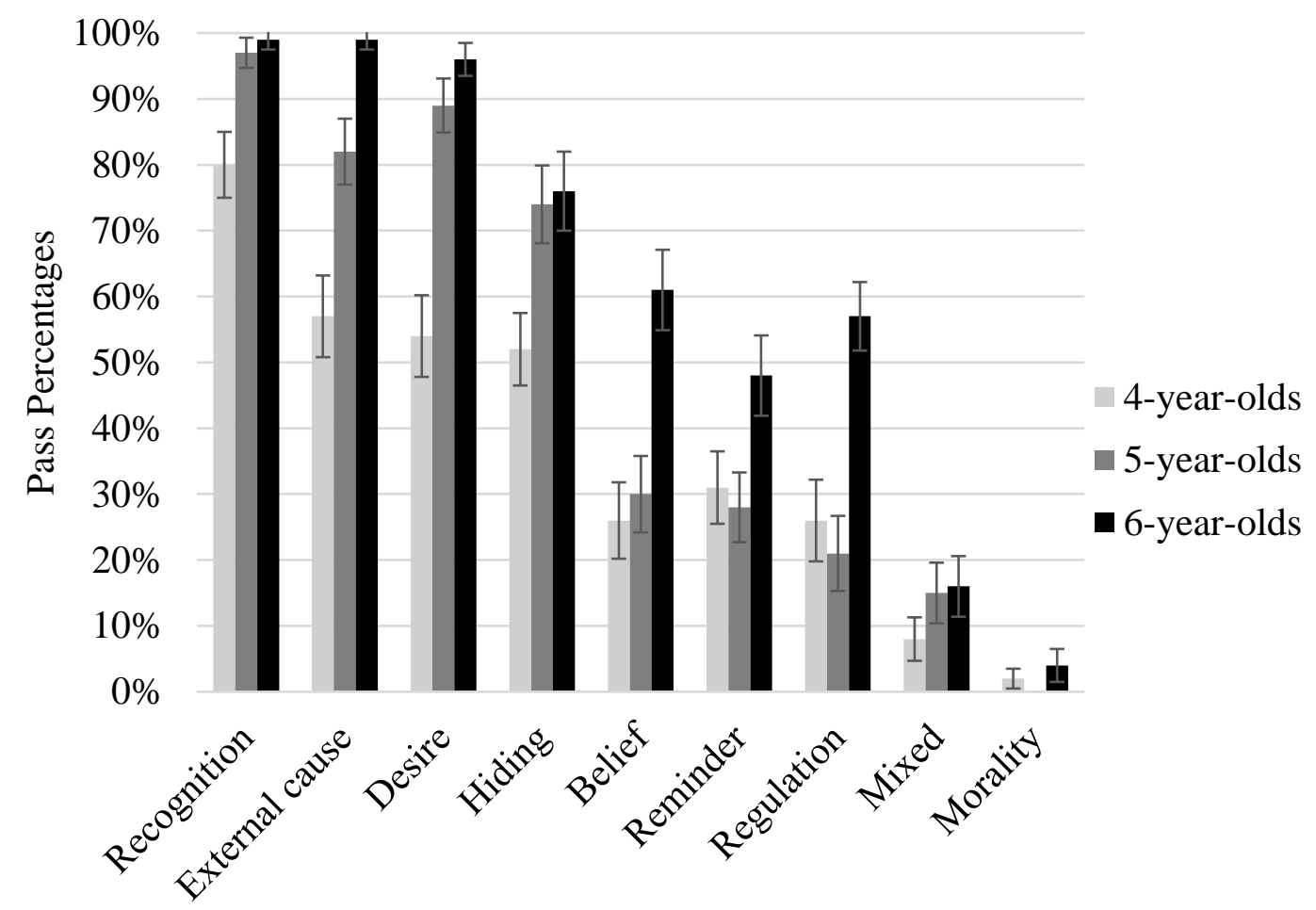

Figure 1. The percentage of children passing each component by age 


\begin{tabular}{|c|c|c|c|c|c|c|c|c|}
\hline \multirow[t]{2}{*}{ Component } & \multicolumn{2}{|c|}{$\begin{array}{c}\text { Chinese } \\
\mathrm{N}=192 \\
\text { Age 4-6 } \\
\mathrm{M}_{\text {age }}=5.19\end{array}$} & \multicolumn{2}{|c|}{$\begin{array}{c}\text { British } \\
\mathrm{N}=60 \\
\text { Age } 3-7 \\
\mathrm{M}_{\text {age }}=5.03\end{array}$} & \multicolumn{2}{|c|}{$\begin{array}{c}\text { German } \\
\mathrm{N}=108 \\
\text { Age 3-6 } \\
\mathrm{M}_{\text {age }}=4.79 \\
\end{array}$} & \multicolumn{2}{|c|}{$\begin{array}{c}\text { Italian } \\
\mathrm{N}=114 \\
\text { Age } 3-6 \\
\mathrm{M}_{\text {age }}=5.03\end{array}$} \\
\hline & Rank & $\begin{array}{c}\text { Pass } \\
(\%) \\
\end{array}$ & Rank & $\begin{array}{c}\text { Pass } \\
(\%) \\
\end{array}$ & Rank & $\begin{array}{c}\text { Pass } \\
(\%)\end{array}$ & Rank & $\begin{array}{l}\text { Pass } \\
(\%) \\
\end{array}$ \\
\hline $\begin{array}{l}\text { I } \\
\text { (Recognition) }\end{array}$ & 1 & 91.7 & 1 & 73.3 & 1 & 75.9 & 1 & 71.9 \\
\hline $\begin{array}{l}\text { II } \\
\text { (External) }\end{array}$ & 2.5 & 79.2 & 3 & 65.0 & 2 & 56.5 & 2 & 50.0 \\
\hline $\begin{array}{l}\text { III } \\
\text { (Desire) }\end{array}$ & 2.5 & 79.2 & 4 & 50.0 & 3 & 44.4 & 4 & 43.0 \\
\hline $\begin{array}{l}\text { IV } \\
\text { (Belief) }\end{array}$ & 5 & 39.6 & 5 & 48.3 & 6 & 27.8 & 6 & 36.8 \\
\hline $\begin{array}{l}\mathrm{V} \\
\text { (Reminder) }\end{array}$ & 6 & 35.9 & 2 & 68.3 & 4 & 35.2 & 5 & 42.1 \\
\hline $\begin{array}{l}\text { VI } \\
\text { (Regulation) }\end{array}$ & 7 & 35.4 & 7 & 18.3 & 7.5 & 26.9 & 8 & 29.8 \\
\hline $\begin{array}{l}\text { VII } \\
\text { (Hiding) }\end{array}$ & 4 & 67.2 & 6 & 40.0 & 5 & 29.6 & 3 & 48.2 \\
\hline $\begin{array}{l}\text { VIII } \\
\text { (Mixed) }\end{array}$ & 8 & 13.0 & 8 & 13.3 & 9 & 10.2 & 9 & 13.2 \\
\hline $\begin{array}{l}\text { IX } \\
\text { (Moral) }\end{array}$ & 9 & 2.1 & 9 & 10.0 & 7.5 & 26.9 & 7 & 35.1 \\
\hline
\end{tabular}

Table 1. Percentage correct and rank of each component of the TEC for Chinese,

British, German, and Italian children

Tang, Y., Harris, P., Pons, F., Zou, H., Zhang, W., \& Xu, Q. (in press). The Understanding of Emotion among Young Chinese Children. International Journal of Behavioural Development. 


\begin{tabular}{lcccc}
\hline \multicolumn{1}{c}{1} & 2 & 3 & 4 \\
\hline 1. Age & - & & & \\
2. TEC score & $0.58^{* *}$ & - & $0.25^{* *}$ & $0.26^{* *}$ \\
3. Verbal ability & $0.60^{* *}$ & $0.51^{* *}$ & - & $0.28^{* *}$ \\
4. Theory of Mind & $0.46^{* *}$ & $0.45^{* *}$ & $0.47^{* *}$ & - \\
$\quad M$ & 5.19 & 4.43 & 12.56 & 3.34 \\
$\quad S D$ & 0.93 & 1.61 & 6.37 & 1.24 \\
\hline
\end{tabular}

Note: ${ }^{* *} p<0.01$

Table 2. Person's correlation coefficients among the TEC scores, verbal ability, theory of mind, and age, with both no control for age (under the diagonal) and with a control for age (above the diagonal) 